\title{
Selective Testing of At-Risk Blood Donors for Trypanosoma cruzi and Plasmodium spp. in Switzerland
}

\author{
Christoph Niederhauser $^{a} \quad J^{\prime}$ ochen Gottschalk ${ }^{b}$ \\ a Interregional Blood Transfusion Service SRC, Berne, Switzerland; \\ ${ }^{b}$ Blood Transfusion Service SRC Zürich, Schlieren, Switzerland
}

\author{
Caroline Tinguely ${ }^{a}$
}

\section{Introduction}

Selective testing - Chagas - Malaria - Trypanosoma cruzi . Plasmodium spp.

\section{Summary}

Background: Population migrations and overseas recreational travel to regions at risk for tropical diseases are increasing. A major challenge in non-endemic countries is to decrease the number of blood donor deferrals due those tropical disease pathogens, without compromising the high level of blood safety. The protozoans Trypanosoma cruzi and Plasmodium spp., the causative organisms of Chagas disease (CD) and malaria are becoming a major focus in the blood transfusion community. Methods: National guidelines of the Blood Transfusion Service of the Swiss Red Cross propose an algorithm for dealing with these pathogens, including a mandatory selective serological testing of donors at risk. Results: 6,978 donors at risk for CD were tested. Three of them were confirmed anti-T. cruzi-positive, and in one case a transfusion-transmitted infection was highly possible. The specificity of the assay was $99.94 \%$. For malaria 12,887 donors were at risk and 178 were confirmed positive. The specificity of the assays was $92.8 \%$. Conclusion: $\mathrm{CD}$ and malaria in non-endemic countries may represent a certain risk for blood transfusion. Switzerland chose a selective testing approach. The specificity of the assays is a crucial topic for this approach because it ensures a minimal loss of false-reactive donors and helps towards an easier counselling of implicated donors.
During the last decade, two emerging pathogens, the protozoan parasites Trypanosoma cruzi and Plasmodium spp., the causative organisms of the Chagas disease (CD) and malaria have become a major focus in the blood transfusion community. Trypanosoma cruzi infections are most commonly transmitted by infected faeces after the bite of blood-sucking triatomine bugs Rhodnius prolixus and Triatoma infestans [1]. CD is widely distributed on the American continent, especially in Latin America, where it is estimated that 25 million people live in risk areas. Worldwide 8-10 million individuals are suspected to be infected, with the majority living in Latin America [2]. Every year $>40,000$ new CD infections are registered worldwide. For many years $\mathrm{CD}$ was restricted geographically to Latin America but due to the increased migratory flow of millions of individuals during recent decades the traditional epidemiological pattern of CD has dramatically changed. Congenital as well as transfusion-related CD cases were reported in many European countries where effective control measures and preparedness plans were not yet established [3, 4]. A recent comprehensive estimation of affected European populations reported that between 14,000 and 180,000 individuals are infected with T. cruzi [5]. The average prevalence of T. cruzi infections in Europe is 35 cases per 100,000 inhabitants; however, a great variation is observed between countries. Whereas in Eastern Europe it is practicably unknown, in Spain 307 cases per 100,000 inhabitants were reported. Piron and co-workers [3] estimated that between 39,985 and 65,258 individuals living in Spain, but of Latin American origin, are infected with T. cruzi. In Italy, Sweden, Portugal and Switzerland, the average number of CD cases per 100,000 inhabitants lies between 25 and 28 [5].

After the initial infection, a life-long chronic persistence of the parasite is often established, with detectable antibody and intermittent parasitaemia. Up to $30 \%$ of infected individuals will develop chronic $\mathrm{CD}$ affecting the smooth muscle of the heart or their gastrointestinal tract $20-30$ years post infection. 
The probability of transfusion-transmitted T. cruzi infections depends on many factors. These include: i) the amount of blood transfused, ii) the infectivity of the parasite contaminating the blood component, iii) the parasite strain, iv) the level of parasitaemia at the time of donation, $v$ ) the immune status of the recipient and vi) the efficiency of the screening assay used [6,7]. Several transfusion-transmitted CD cases have been observed since 1952 [8]. During the last decades between 300 and 800 cases are estimated $[9,10]$. As a consequence of this estimation, many countries established successful T. cruzi antibody blood-screening programmes, and these have helped to reduce the occurrence of transfusion-transmitted T. cruzi infections.

The relevance of transfusion as a route of $T$. cruzi transmission is due to the existence of an asymptomatic but parasitaemic chronic phase of the disease as well as the $\mathrm{CD}$ prevalence in various populations. Blood transmission of T. cruzi may occur primarily because potential blood donors are frequently unaware of their infection status [11]. A chronic asymptomatic carrier state in conjunction with an asymptomatic or non-specific clinical infection course in recipients of T. cruzi-infected blood results in transfusion-transmission cases being undetected and thus unreported. Transfusion-based T. cruzi infections are already a major route of transmission in many non-endemic countries, with several cases reported recently in USA, Canada, Spain and Mexico [3, 4, 12]. It is estimated that on average $20 \%$ (range $0-40 \%$ ) of the transfusion recipients of parasitaemic blood became infected [13-15]. All of these cases involved either pooled or apheresis platelet concentrates (PCs). Irradiation or leucocyte reduction procedures appear not to provide much protection against these pathogens.

A chronic phase low-level parasitaemia is observed in 50\% of those individuals infected [16]. There is often less than one trypanosome circulating in $20 \mathrm{ml}$ of peripheral whole blood in chronically infected individuals. This extremely low level of parasitaemia makes even individual NAT strategies insufficient to prevent $T$. cruzi infections from chronically infected blood donors [9]. Thus direct methods to detect the parasite are not advised. T. cruzi antibodies on the other hand appear 4-8 weeks post infection and persist unless the parasite is eradicated [11]. Serological testing is thus the method of choice to detect T. cruzi-infected blood donations in non-endemic countries.

Governmental health policies to prevent transfusion of T. cruziinfected blood components vary for endemic and non-endemic countries. In endemic countries all donations should be analysed for T. cruzi antibodies [6]. In countries where the number of infectious donors is substantially lower several different strategies are envisaged such as i) a permanent deferral of at-risk donors, ii) a universal donor screening, iii) selective one-time donor screening or iv) selective donor screening including analysis of a special donor questionnaire [17]. The most common strategy established is selective testing, and this is already implemented in several nonendemic countries [18, 19].

The use of a specific questionnaire to detect at-risk donations is surprisingly effective as was demonstrated by investigations conducted in Canada [20]. The effectiveness of a selective testing ap- proach however does rely mainly upon the ability and the willingness of the donor to correctly answer questions of suspected risk situations. In a study conducted in the USA only $75 \%$ of the infected donors were identified via the questionnaire [12]. Thus although $100 \%$ safety will never be achieved, performing a selective testing in parallel with a specific questionnaire should detect the majority of infections.

Currently 35,000 registered inhabitants of Switzerland originate from a T. cruzi-endemic region in Latin America, and this figure increases when also unregistered migrants are included. During a routine serological screen of Swiss blood donors conducted in 2009 for malaria, an initial anti-Plasmodium spp.-reactive enzyme immunoassay (EIA) sample turned out to be anti-T. cruzi EIA-positive after further investigations. It was from a repeat donor who originated from Colombia and had previously given 3 donations. Post-donation samples could only be collected from 1 patient, but this was serologically T. cruzi-negative. A pilot EIA study performed among 1,012 Latin American migrants living in Geneva showed that $\mathrm{CD}$ is highly prevalent among these individuals, especially in those from Bolivia. Overall $12.8 \%$ were anti-T. cruzi-positive, with $\geq 26.2 \%$ in the Bolivian subpopulation. All positive individuals were in a chronic phase of the infection. This study also highlighted that these individuals were considering donating blood [21]. Based on this observation, a voluntary T. cruzi EIA screening study was conducted on some at-risk donors in two regions of Switzerland. Based on the results of these studies, the Blood Transfusion Service of the Swiss Red Cross decided in January 2013 to introduce T. cruzi-selective testing as mandatory for donors being at risk for T. cruzi infection [22].

Malaria, a protozoan parasitic infection of humans, is occasionally a fatal disease. The infection is caused by one or more of the five species of the genus Plasmodium (P. falciparum, P. vivax, P. ovale, P. malariae, P. knowlesi) [23]. The malaria parasite is most commonly transmitted by bites from infected Anopheles mosquitoes. Worldwide, it is estimated there are approximately 207 million acute cases of malaria every year, with $80 \%$ or more occurring in Africa [24]. Each year, $>1$ million people die from malaria [25, 26].

Malaria can also be transmitted by blood components and transfusion-transmitted malaria cases (TTM), although rare, continue to pose a risk to blood services worldwide [27]. Several of the most recently reported TTM cases result from 'semi-immune' donors who carry undetectable levels of parasites in their blood without clinical symptoms [28-32]. TTM is an important clinical and public health problem, especially in populations with no immunity to malaria where it can be rapidly fatal if not recognised and quickly treated [33]. P. falciparum infection in non-immune individuals was reported to be fatal in up to $10 \%$ of patients infected [34]. Malaria poses a problem in many European countries where a considerable proportion of the blood transfusion recipients are immunocompromised. Furthermore, increasing numbers of potential donors have a background from a malaria-endemic area.

According to the current European regulations, it is mandatory that donors who present with risk of malaria are deferred for 6 months to 3 years, depending on the severity of their exposure risk 


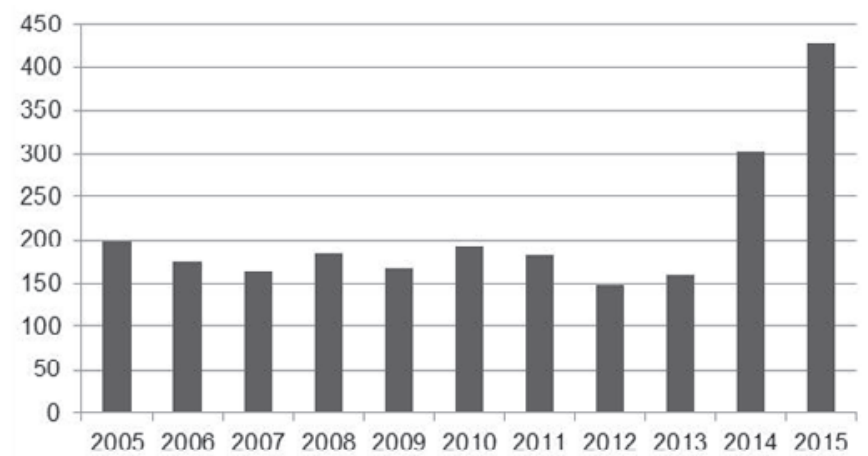

Fig. 1. Notified malaria cases per year in Switzerland form 2005 until 2015.

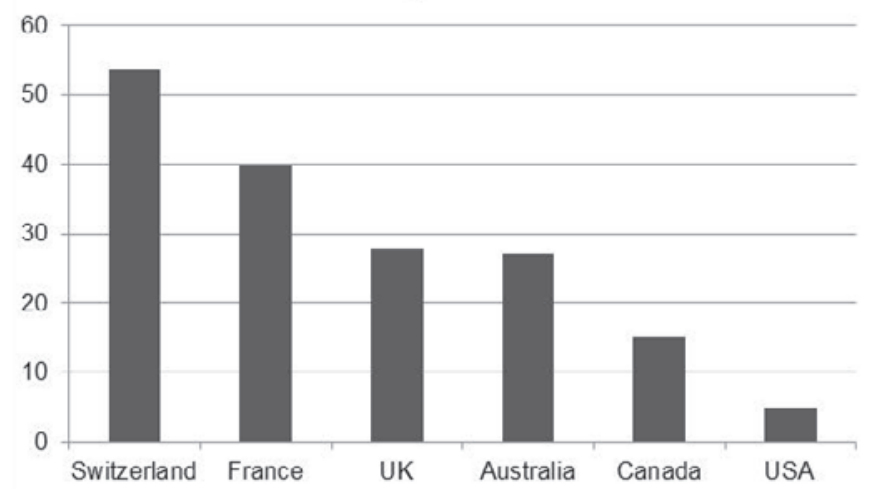

Fig. 2. Comparison of notified malaria cases per million inhabitants from different developed countries. to the Plasmodium parasite. Many western countries, including France, UK, Australia, Denmark, Finland, New Zealand and Switzerland, have implemented selective malaria screening programmes during the last 20 years $[32,35-37]$. This policy has been very successful as no or only very few TTM infections have been detected since then. The USA and Canada rely solely on a deferral strategy to prevent recipients from possible TTM $[38,39]$. Other countries, including Norway and Spain, have a deferral strategy supplemented with selective serological testing to reduce the deferral time or to accept new donors [36]. In summary, the consensus of the different strategies is to defer donors for long enough after their travel to, or residence in, a malaria-endemic region to allow them to develop symptoms or resolve their infection [36]. Shorter deferral periods are allowed if the donors are screened serologically.

On average 200 cases of imported malaria infections are diagnosed in Switzerland every year. The majority of these cases originated in Africa (75\%), followed by Asia and America (www.bag. admin.ch/themen/medizin/00682/00684/01086/index.html?lang = de). Since 2013, however, there has been a great increase in the number of notified malaria cases. In 2014 and 2015, 303 and 428 cases respectively were reported (fig. 1). Up to 2013, the incidence of imported malaria cases in Switzerland was similar to that observed in other European countries, Australia and Canada [19]. The increase observed in Switzerland since 2014 has not been noticed in other countries (fig. 2).

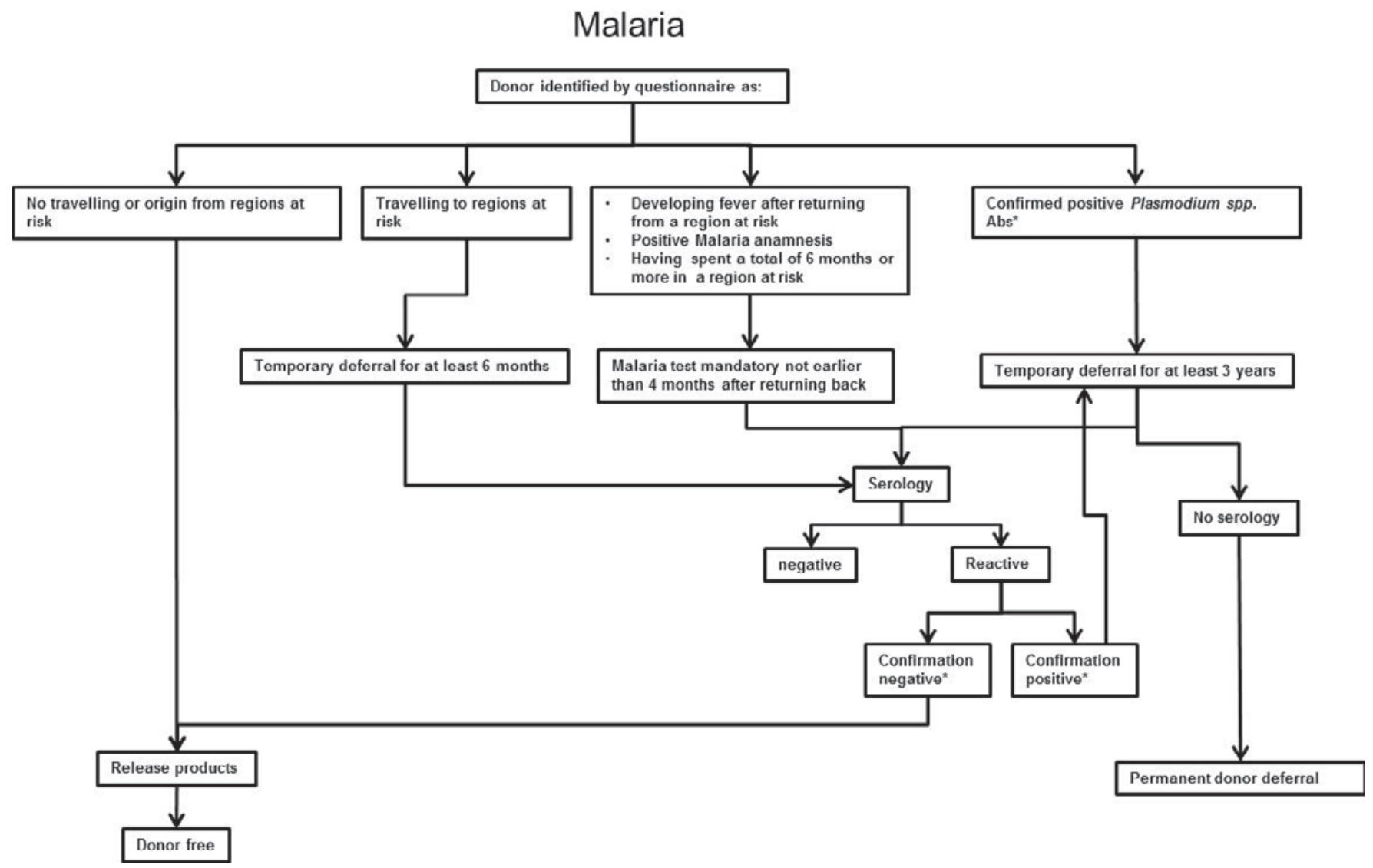

*Confirmation performed at the Swiss Tropical Institute.

Fig. 3. Algorithm for donors at risk for malaria. 


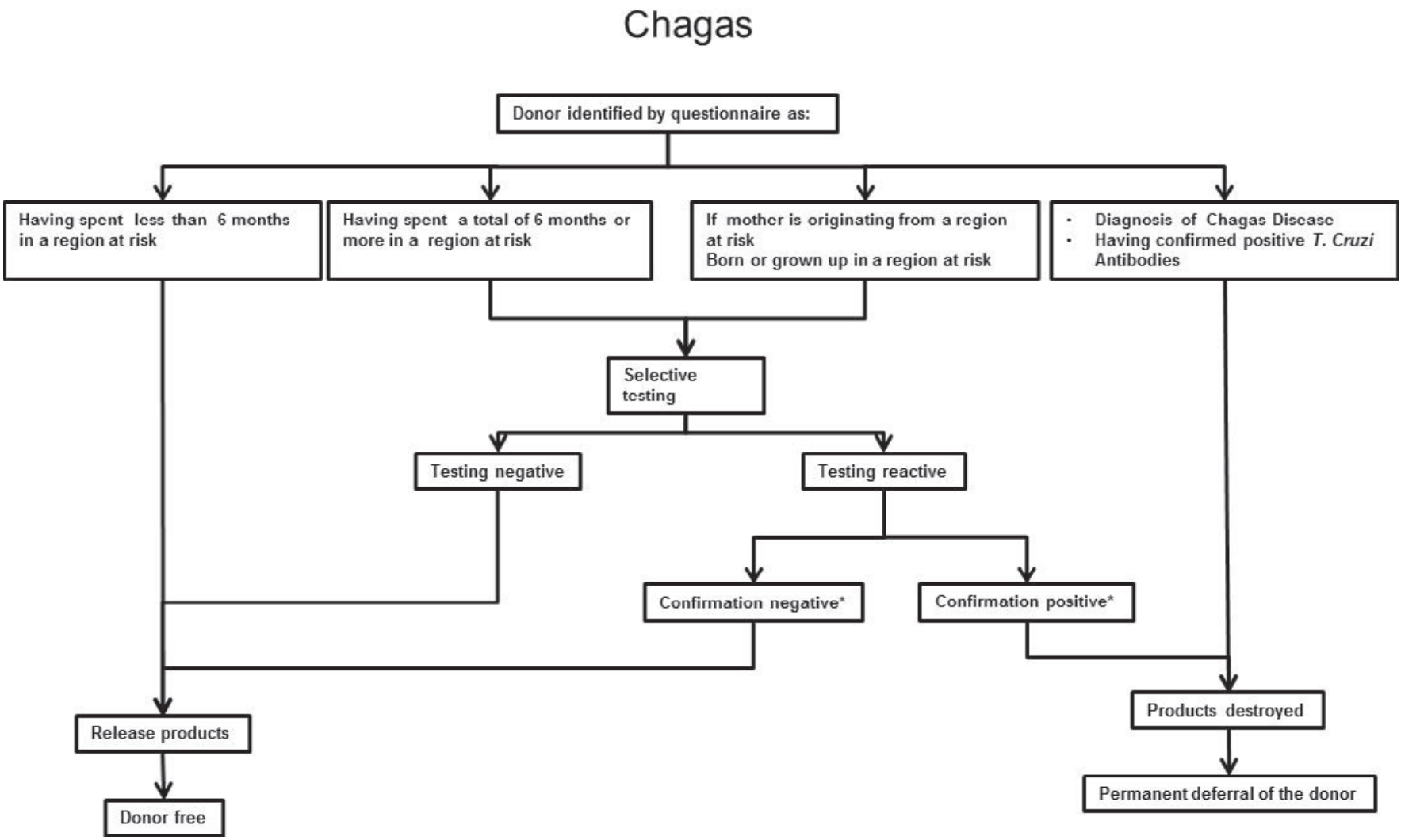

*Confirmation performed at the Swiss Tropical Institute.

Fig. 4. Algorithm for donors at-risk for Chagas disease.

Selective anti-Plasmodium spp. testing was introduced in April 2007 in Switzerland for those donors directly originating from or who had stayed longer than 6 months in a malaria-endemic region (fig. 4). There have only been rare cases of TTM cases reported in Switzerland, but TTM is still regarded a serious concern for the reasons mentioned previously. The last fatal Swiss TTM case was reported in 1999, several years before regulated screening programmes were implemented [28].

The selective testing data collected for the Swiss blood donor population at risk are reported for both parasitic diseases $\mathrm{CD}$ and malaria.

\section{Material and Methods}

The national guidelines of BTS SRC drew up an algorithm for dealing with donors at risk for CD. This is presented schematically in figure 4. Testing for $T$. cruzi antibodies with an EIA or immunofluorescence (IF) test is mandatory for those donors i) directly originating from South or Central America, ii) whose mothers originated from these countries and iii) who have travelled during $\geq 6$ months in a CD-endemic country. These donors were tested with the Chagas CMIA test system (Abbott, Delkenheim, Germany). Initially reactive screening samples were confirmed with an ELISA and IF antibody test in-house assay $(P$. falciparum antigens or culture) at the Swiss Tropical and Public Health Institute.

Figure 3 schematically describes the algorithm proposed by the BTS SRC for dealing with donors at risk for malaria. In Switzerland, donors traveling to regions at risk for malaria are deferred from donating blood for 6 months after their return. Those donors suffering from a fever of unknown origin or other clinical symptoms, those who had positive malaria anamnesis or those who were born or stayed longer than 6 month in a region at risk for malaria are only allowed to donate blood under the prerequisite that they have tested negative for Plasmodium spp. antibodies after a period $\geq 4$ months after their return. These donors are actually screened with an EIA malaria test (Biorad, Cressier, Switzerland). Before December 2011, it was an in-house assay from the Institute of Parasitology, Zurich). Both assays detect antibodies which appear at all stages of infection and against all four species known to cause human malaria - P. falciparum, $P$. vivax, $P$. ovale and $P$. malariae - but no distinction can be made between the species. A special post-donation questionnaire was used to collect additional information, including age, sex, and length of stay in region at risk, prophylaxis, symptoms, diagnosis and possible treatment from donors with confirmed positive malaria antibodies.

\section{Results}

\section{Chagas Disease}

Between March 2011 and December 2012, around 650,000 blood donations were collected in Switzerland. In a pilot project approximately 50,000 of the donors were asked if they had a risk of $\mathrm{CD}$, and those who gave a positive reply were tested with an anti- $T$. cruzi EIA. In total 729 donors were tested, but all were negative for T. cruzi antibodies. A mandatory, selective anti-T. cruzi testing algorithm was introduced in January 2013 for all donors at risk for CD. Between January 2013 and December 2015, approximately 1.1 million individuals donated blood in Switzerland. From these donors, $6,978(0.63 \%)$ were designated at risk for $\mathrm{CD}$ and tested for $T$. 
cruzi. The vast majority were negative for T. cruzi antibodies. Three of the 6,978 donors were confirmed positive for $\mathrm{CD}$, a further 3 turned out to be false-reactive, and 1 donor was screening indeterminate but negative in confirmation. The specificity of the assays was $99.94 \%$. The overall frequency of T. cruzi antibodies for donors at risk for CD was 1:2,326, including donations collected during the pilot phase.

From a selection of those donors at risk for $\mathrm{CD}$, a questionnaire relating to their risk behaviour was evaluated. Of the total 1,639 donors assessed, 649 (39.6\%) were born and grew up in an affected region, 485 (29.6\%) had mothers originating from at-risk areas, and $505(30.8 \%)$ had travelled longer than 6 months in South and/ or Central America.

Look-back procedures were initiated for the $3 \mathrm{~T}$. cruzi-positive donors. The first was a 32-year-old male first-time donor who had lived several years in South America. The blood components were destroyed, thus no look-back procedure was initiated. The two other implicated donors were repeat donors. One male donor who was born in 1950 and grew up in Bolivia had donated 3 times. From his first two donations 2 red blood cell (RBC) concentrates were transfused to different patients. The plasmas were sent for fractionation. Only 1 of the 2 recipients of the these RBC concentrates was eligible for a blood drawing. His post-transfusion sample was negative for T. cruzi antibodies. The second repeat donor, originated from Brazil, had immigrated to Switzerland in 1973 and had donated 54 times since 1995 [40, 41]. From these donations 77 blood components were produced including $22 \mathrm{RBC}$ concentrates, 27 PCs, which were predominately not pathogen-reduced, 12 fresh frozen plasma (FFP) units and 16 units of plasma for fractionation (FPI). A look-back procedure was initiated, but the response from the hospitals was unfortunately quite poor. A feedback was obtained from only 16 recipients of the 77 products. Most of the involved patients have died from their primary disease, and therefore no post-transfusion samples were available. Nevertheless, a 70 year-old patient who had received a non-pathogen reduced PC in 2008 could be identified. Following kidney transplantation, the patient was hospitalised in 2010 due to fever, cough, dyspnoea and increasing kidney failure. Shortly afterwards the patient died having suffered a cardiogenic shock and multi-organ failure. The autopsy revealed a generalised myocarditis with a distinct infiltration of T. cruzi.

\section{Malaria}

From the 3.15 million blood donations collected in Switzerland between April 2007 and December 2015, 12,887 were screened for antibodies against Plasmodium spp. (0.41\%). The majority were negative $(11,724)$, but 1,011 were reactive and a further 152 fell within the grey zone of the assay. Theses samples were sent to the Swiss Tropical Institute (STI) for additional test confirmation. From the 1,163 donors, 178 were confirmed positive. This results in a rate of 1:17,697 Plasmodium spp.-confirmed donations. The specificity of the malaria EIA was thus $92.8 \%$. Prior to 2013 , no mandatory confirmation was in place, and the 237 screening-reactive samples from this period were not confirmed by additional testing.
Many of the donors which were subsequently confirmed malaria-positive had also completed a post-donation questionnaire regarding their country of origin or regarding the countries they had visited during their travels. The origin of 154 of these donors was determined to be an endemic country: 143 from Africa (Cameroun (37), Ivory Coast (23), Togo (11), Congo (11), Nigeria, Benin, Kenya and others), 9 from Asia (Sri Lanka, India, Thailand and Vietnam) and 2 from South America (Brazil and Peru). Thus the vast majority of malaria-positive donors (150) originated from African and Asian countries, whereas only 4 donors had extensively travelled in malaria-endemic areas (1 in Malaysia, 1 in Tanzania, 2 unknown). For the remaining confirmed positive donors, their status could not be documented. Most of the donors originating from an endemic country recalled having symptoms of malaria in their childhood or youth without receiving adequate prophylaxis. But 25 donors who had never taken malaria prophylaxis as well as 5 donors who had received prophylaxis did not recall having any malaria symptoms. Only 4 donors remembered having malaria symptoms in spite of prophylaxis.

\section{Discussion}

Many tropical diseases, including CD and malaria, have become a major focus for the producers of blood components during the last $10-15$ years, primarily due to increased population migrations and overseas recreational travel to regions at risk for tropical diseases. A major challenge in non-tropical disease-endemic countries is to decrease the number of donor deferrals due to $\mathrm{CD}$ or malaria without compromising the high level of blood safety. There are different envisaged approaches to circumvent this situation. Two commonly used strategies, permanent deferral and selective testing, rely mainly on the specific questionnaire to identify a donor at risk. In turn, the effectiveness of a questionnaire depends on the ability of donors to correctly answer risk questions.

Current deferral rates for at-risk behaviour suggest most donors acknowledge their risk; however, it is well documented that a few donors with transmissible infections do fail for one reason or another to declare their risk [42-45]. Inaccurate information may be communicated intentionally or unintentionally either because they misunderstood the questions asked or because they were unaware or have forgotten certain risk behaviour [29]. The simplest approach taken by many countries is thus to identify and defer all potential donors at risk for $\mathrm{CD}$ and malaria. However, the loss of donors deferred due to a risk of CD or malaria is often quite significant, and thus only few countries can afford to lose so many donors.

Because Plasmodium spp. and T. cruzi are expected to be rare imported infections in Switzerland, it was assumed that antibodypositive donors would be most efficiently identified by questioning the donors and a subsequent selective serological testing approach. This policy is similar to that employed in other low-endemic countries, including Canada, UK and France [19]. The current EU directives 2004/33/CE specifies active CD as a blood donor exclusion 
criterion, but unfortunately does not recommend adequate measures to adopt for those donors exposed to CD but have not yet been screened. Enforced since January 2013, the Swiss health authorities prescribed a CD-selective testing approach for at-risk donors in order to release blood components. Only $0.63 \%$ and $0.41 \%$ of the donations originated from donors at-risk for $\mathrm{CD}$ and malaria respectively. Only very few donations subsequently confirmed positive: $3 \mathrm{CD}$-positive from 6,978 donations and 178 malaria-positive from 12,887 donations.

The prevalence of blood donors infected with CD in several European countries has been recently published and varies from 1 : 161 in Spain to 1:10,279 in France to 1:12,862 in the UK $[3,18,46]$. The three confirmed positive cases from 6,978 donors $(1: 2,326)$ which were identified in Switzerland lies within the same range but is too low to be statistically significant. Transfusion-transmitted $\mathrm{CD}$ has been often reported, and it has been documented that several different blood components can be involved $[3,4,12]$. The infective capacity of each type of labile blood component varies from 10 to $25 \%$. PCs pose a greater risk than RBC concentrates, frozen plasma or cryoprecipitated products $[4,47-55]$. From the 3 confirmed positive cases from Switzerland, 1 donor was a first-time donor, whereas the 2 others were repeat donors. The initiated lookback procedures for blood components of the 2 repeat donors revealed in 1 case a likely transmission of T. cruzi to a recipient. The look-back procedures were, as is often the case, not always successful. The limited feedback from the hospitals was probably caused by the large space of time since the implicated blood components were transfused. Many of the recipients had already died from their primary disease, and thus no post-transfusion sample was available. Only $20 \%$ of the requests were replied by the hospitals. The repeat donor responsible for the CD transmission had donated 54 times, and 77 components were produced. A 70-year-old patient who had received a non-pathogen reduced PC in 2008 was identified. Shortly afterwards the patient died having a cardiogenic shock and multi-organ failure, and the autopsy conducted revealed a generalised myocarditis with infiltration of T. cruzi [40, 41].

Recent pathogen reduction technologies with amotosalen, in conjunction with UV illumination, can reduce the pathogens $>5$ $\log$ [56-58]. The mandatory Intercept ${ }^{\circledR}$ pathogen procedure for apheresis and pooled PCs, which was introduced in Switzerland in July 2011, has resulted in a substantial increase of the safety of these components with respect to all pathogens including T. cruzi.

A high specificity of the EIA assays used is essential for a selective testing approach as this should ensure that eligible donors are not deferred due to false-reactive results. In a Canadian study, 59 of 81,616 tested donations were repeat reactive for T. cruzi antibodies, but only one of them was eventually confirmed positive [59]. A specificity of $99.93 \%$ was reported for the EIA used in this study which compares well with the $99.94 \%$ specificity of the assay used in Switzerland.

Between 2006 and 2013, on average of 21.5 malaria cases per million inhabitants were registered by the Swiss health authorities. In several European countries, Australia and Canada, a similar prevalence has been reported [19]. Since 2014, a significant in- crease was observed in Switzerland, with 38 and 53.5 per million inhabitants in 2014 and 2015 respectively, levels at the upper end observed in many other non-endemic countries. The increase was mainly observed in young adult males and to lesser extent females (15-29 years of age) and is most likely caused by the recent increased flow of young migrants from malaria-endemic countries. This increased incidence of malaria cases has not however been seen in the incidence of Plasmodium spp. antibodies in the blood donor population. This is probably due to the exclusion policy setup in Switzerland which does not allow immigrant individuals to donate blood.

Deferral policies of blood donors at risk of transmitting malaria segregate potential donors into two groups: individuals who were born and had lived for several years in malaria-endemic areas and those who were born and resided in non-endemic areas but who have visited an endemic area. The European guidelines for dealing with donors at risk for malaria specify using an immunologic or molecular assay test. If the assay is negative and at least 6 months have elapsed since the visit to an endemic area, the individuals are invited to donate. Many directives, including those from France and UK, allow donors which were deferred for $>4$ months to be retested. Those donors with negative results are released from deferral, while those still reactive are permanently deferred, thus preventing those small number of individuals which harbour persistent low-level parasitaemia from entering the donor pool.

In the UK, 35,000-45,000 blood donations are collected yearly by NHS Blood and Transplant from donors at risk for malaria, and about $20 \%$ of these donors declare residency as their risk [18]. This picture may vary in other non-endemic countries depending on the origin of immigrants and traveller pattern. Travellers, though numerically the largest group of donors at risk for malaria, present a much lower risk than those donors who were either born in or declare a previous residency in a malaria-endemic area. The majority of individuals with reactive screening results demonstrate a serological response to past resolved infection; a small number may still be infected with a persistent low-level parasitaemia. These donors are likely to be those categorised as 'semi-immune' individuals with high-titre antibody and persistent low-level parasitaemia. Such individuals often originate from endemic areas with particularly high levels of malaria infection where frequent exposure to malaria parasites occurs, leading to a dynamic balance between the infection and the immune response. Since the implementation of the selective testing approach for donors being at risk for malaria in Switzerland, 178 were found to be confirmed positive for Plasmodium spp. antibodies, with the vast majority of these donors originating from an endemic country. Only 4 donors were of European origin and had travelled in endemic areas. Though the greatest risk comes from semi-immune former residents of endemic areas, the greatest impact on sufficiency (donor loss) comes from low-risk short-term travelling donors. This scenario suggests that a selective testing approach is the most logical approach. France was the first country to implement selective testing in 1986 [32]. Other countries (UK, Australia, Denmark and others) successively introduced similar testing form 2001 onwards [35, 36, 60]. In Switzer- 
land selective testing for anti-Plasmodium antibodies has been mandatory since April 2007.

Very few cases of transfusion-transmitted malaria have been reported in developed countries since 2002, and all have lived for an extensive period in Africa and had reported recent travel history to this region [19]. The last case reported in Switzerland was even further back in 1999 - also an individual from Africa with a travel history.

The percentage of potential donors screened for Plasmodium spp. antibodies $(0.41 \%)$ is much lower than in other countries (USA 1.4\%; 1.8\% UK, 3.1\% Canada, 6.0\% France, 13.5\% Australia) [19]. The repeat reactive rate was on the other hand higher in Switzerland $(8 \%)$ than in other countries $(1.8-5.47 \%)[19,35]$. This highlights the low specificity of the tests used $(92.9 \%$ in Switzerland). This low specificity poses a problem as many initial reactive donations must be confirmed, and if not well managed otherwise eligible donors will be lost. Only 178 of the 1,163 initially reactive or grey-zone donations were confirmed positive. A certain proportion had still an indeterminate confirmation and these were deferred for further 3 years according to the Swiss guidelines.

The selective screening strategy for Plasmodium spp. antibodies implemented since 1999 is effective, and no TTM has been reported in Switzerland since the introduction. In Germany, donors travelling to regions at risk are temporarily deferred for 6 months after their return. According to the German guidelines, donors born or having lived permanently in at-risk regions for at least 6 months must be deferred after immigration for at least 4 years. After this temporary deferral, they are allowed to donate blood if they test negative for Plasmodium spp. antibodies. If no testing could be performed, these individuals are permanently deferred [61]. This approach, though successful, has surely caused the loss of otherwise eligible individuals from the donor pool.

Transfusion-transmitted CD and malaria infections have serious implications for the recipient, and their occurrence impacts definitively on public confidence. Selective testing for antibodies to these parasitic diseases seems to be a cost-efficient approach. It ensures safe blood components, does not burden the budget of the blood transfusion services and causes only a small loss of donors and blood components. The specificity of the assay still needs to be addressed. Whereas the T. cruzi EIA has a very high specificity, it is essential that the specificity of the current Plasmodium spp. EIA is increased. Apart from ensuring a minimal loss of false-reactive donors, it will lead to an easier counselling of the implicated donors. In conclusion, the questionnaire and selected testing for T. cruzi and Plasmodium spp. in conjunction with other measures, such as leucocyte reduction and pathogen reduction technologies, will prevent future transfusion-transmitted $\mathrm{CD}$ and malaria infections in non-endemic countries.

\section{Acknowledgements}

Dr. med. Laura Infanti for investigating two CD look-backs and Dr. phil. nat. Peter Gowland for editing the manuscript.

\section{Disclosure Statement}

The authors declare no conflict of interest for this article.

\section{References}

1 Rassi A Jr, Rassi A, Marcondes de Rezende J: American trypanosomiasis (Chagas disease). Infect Dis Clin North Am 2012;26:275-291.

2 World Health Organization: First WHO Report On Neglected Tropical Diseases: Working of Overcome the Global impact of Neglected Tropical Diseases (WHO/ HTM/NTD/2010.1) 2010 (cited 2012 March 20); Available from http://whqlibdoc.who.int/publications/2010/ 9789241564090 eng.pdf (last accessed April 19, 2016).

3 Piron M, Vergés M, Muñoz J, Casamitjana N, Sanz S, Maymó RM, Hernández JM, Puig L, Portús M, Gascón J, Sauleda S: Seroprevalence of Trypanosoma cruzi infection in at-risk blood donors in Catalonia (Spain). Transfusion 2008;48:1862-1868.

4 Benjamin RJ, Stramer SL, Leiby DA, Dodd RY, Fearon M, Castro E: Trypanosma cruzi infection in North America and Spain: evidence in support of transfusion transmission. Transfusion 2012;52:1913-1921.

5 Strasen J, Williams T, Ertl G, Zoller T, Stich A, Ritter O: Epidemiology of Chagas disease in Europe: many calculations, little knowledge. Clin Res Cardiol 2014; 1031:1-10.

6 Schmunis GA: Epidemiology of Chagas disease in nonendemic countries: the role of international migration. Mem Inst Oswaldo Cruz 2007;102(suppl 1):75-85

7 Wendel S, Gonzaga AL: Chagas' disease and blood transfusion: a New World problem? Vox Sang 1993;64: $1-12$.
$D_{8}$ Pedreira De Freitas JL, Amato Neto V, Sonntag R, Biancalana A, Nussenzveig V, Barreto JG: First tests on the accidental transmission of Chagas disease to man by blood transfusion. Rev Paul Med 1952;40:36-40.

$\checkmark$ Wendel S: Transfusion-transmitted Chagas' disease. Curr Opin Hematol 1998;5:406-411.

10 Hernandez-Becerril N, Mejia AM, Ballinas-Verdugo MA, Garza-Murillo V, Manilla-Toquero E, Lopez R, Trevethan S, Cardenas M, Reyes PA, Hirayama K, Monteon VM: Blood transfusion and iatrogenic risks in Mexico City. Anti- Trypanosma cruzi seroprevalence in 43,048 blood donor, evaluation of parasitemia and electrocardiogram findings in seropositive. Mem Inst Oswaldo Cruz 2005; 100:111-116.

11 Castro E: Chagas disease: lessons from routine donations testing. Transfus Med 2009;19:16-23.

12 Menitove JE, Bennett JL: Lessons learned from Trypansoma cruzi test implementation. Transfusion 2012; 52:1849-1851.

13 Schmunis GA: Prevention of transfusional Trypanosoma cruzi infection in Latin America. Mem Inst Oswaldo Cruz 1999;94:93-101.

14 Leiby DA, Herron RM, Read BA, Lenes BA, Stumpf RJ: Trypanosoma cruzi infection in Los Angeles and Miami blood donors: impact of evolving donor demographics on seroprevalence and implications for transfusion transmission. Transfusion 2002;4:549-555.
15 Kirchhoff LV, Paredes P, Lomeli-Guerrero A, ParedesEspinoza M, Ron-Guerrero CS, Delgado-Mejia M, Pena-Munoz JG: Transfusion-associated Chagas disease (American trypanosomiasis) in Mexico; implications for transfusion medicine in the United States. Transfusion 2006;46:298-304.

16 Schmunis GA: Trypanosoma cruzi, the etiological agent of Chagas disease: status in the blood supply in endemic and nonendemic countries. Transfusion 1991;31:547-557.

17 Angheben A. Boix L, Buonfrate D, Gobbi F, Bisoffi Z, Pupella S, Gandini G, Aprili G: Chagas disease and transfusion medicine : a perspective from a non-endemic countries. Blood Transfus 2015;13:540-550.

18 Kitchen AS, Hewitt PE, Chiodini PL: The early implementation of Trypanosma cruzi antibody screening of donors and donations within England: pre-empting a problem. Transfusion 2012;52:1931-1919.

19 O'Brien SF, Delage G, Seed CR, Pillonel J, Fabra CC, Davison K, Kitchen A, Steele WR, Leiby DA: The epidemiology of imported malaria and transfusion policy in 5 nonendemic countries. Transfus Med Rev 2015; 29:162-171.

20 O’Brien SF, Scalia V, Goldman M, Fan W, Yi QL, Huang M, Ndao M, Fearon MA: Evaluation of selective screening of donors for antibody to Trypanosoma cruzi: seroprevalence of donors who answer 'no' to risk questions. Transfusion 2014;54:863-869. 
21 Jackson Y, Gétaz L, Wolff H, Holst M, Mauris A, Tardin A, Sztajzel J, Besse V, Loutan L, Gaspoz JM, Jannin J, Albajar Vinas P, Luquetti A, Chappuis F: Prevalence, clinical staging and risk for blood-borne transmission of Chagas disease among Latin American migrants in Geneva, Switzerland. PLoS Negl Trop Dis 2010;4:e592.

22 National Swiss Guidelines. https://sbsc-bsd.ch/dokuman/ DokumenteBSD/Allgemeines/tabid/107/Default.aspx (last accessed April 19, 2016).

23 Cullen KA, Arguin PM: Malaria surveillance - United States, 2011 MMWR Surveillance Sum 2013;62(suppl 5):1-17.

24 World Health Organization: World Malaria Report 2013.www.who.int/malaria/publications/world_malaria_ report_2013/report/en/ (last accessed April 19, 2016).

25 Anstey NM, Douglas NM, Poesporodjo JR, Price RN: Plasmodium vivax: clinical spectrum, risk factors and pathogenesis. Adv Parasitol 2012;80:151-201.

26 Douglas NM, Anstey NM, Buffet PA, Poesporodjo JR, Yeo TW, White NJ, Price RN: The anemia of Plasmodium vivax malaria. Malaria J 2012;11:135.

27 Pathak S, Chandrashekhar M: Transfusion transmittable infections - seroprevalence among blood donors in a tertiary care hospital of Dehli. Asian J Transfus Sci 2013;7:116-118.

-28 Frey-Wettstein M, Maier A, Markwalder K, Münch U: A case of transfusion transmitted malaria in Switzerland. Swiss Med Wkly 2001;131:320.

-29 Slinger R, Giulivi A, Bodie-Collins M, Hindieh F, John RS, Sher G, Goldman M, Ricketts M, Kain KC: Transfusion-transmitted malaria in Canada. CMAJ 2001; 164:377-379.

30 Kitchen AD, Barbara JA, Hewitt PE: Documented cases of post-transfusion malaria occurring in England: a review in relation to current and proposed donor-selection guidelines. Vox Sang 2005;89:77-80.

31 Kitchen AD, Chiodini PL: Malaria and blood transfusion. Vox Sang 2006;90:77-84.

32 Garraud O, Assal A, Pelletier B, Danic B, Kerleguer A, David B, Joussemet M, de Micco P: Overview of revised measures to prevent malaria transmission by blood transfusion in France. Vox Sang 2008;95:226-231.

33 Owusu-Ofori AK, Betson M, Parry CM, Stothard JR, Bates I: Transfusion-transmitted malaria in Ghana. Clin Infect Dis 2013;56:1735-1741.

34 Mungai M, Tegtmeier G, Chamberland M, Parise M: Transfusion-transmitted malaria in the United States from 1963 through 1999. N Engl J Med 2001;344: 1973-1978.

35 Kitchen AD, Lowe PH, Lalloo K, Chiodini PL: Evaluation of a malarial antibody assay for use in the screening of blood and tissue products for clinical use. Vox Sang 2004;87:150-155.

-36 Reesink HW, Panzer S, Wendel S, Levi JE, Ullum H, Ekblom-Kullberg S, Seifried E, Schmidt M, Shinar E, Prati D, Berzuini A, Ghosh S, Flesland Ø, Jeansson S, Zhiburt E, Piron M, Sauleda S, Ekermo B, Eglin R, Kitchen A, Dodd RY, Leiby DA, Katz LM, Kleinman S: The use of malaria antibody tests in the prevention of transfusion-transmitted malaria. Vox Sang 2010;98: 468-478.
37 Seed CR, Kee G, Wong T, Law M, Ismay S: Assessing the safety and efficacy of a test-based, targeted donor screening strategy to minimize transfusion transmitted malaria. Vox Sang 2010;98:e182-192.

38 Leiby DA, Nguyen ML, Notari EP: Impact of donor deferrals for malaria on blood availability in the United States. Transfusion 2008;48:2222-2228.

39 O'Brien SF, Uzicanin S, Choquet K, Yi QL, Fan W, Goldman M: Impact of changes to policy for Mexican risk travel on Canadian blood donor deferrals. Blood Transfus 2013;11:580-584.

40 Markovic A, Ries J, Gottschalk J, Glauser A, Frey BM: Status report: lookback on transfused products of a $T$. cruzi positive blood donor. Clin Lab 2014;8:6.

41 Ries J, Komarek A, Gottschalk J, Brand B, Amsler L, Frey BM: A case of possible Chagas transmission by blood transfusion in Switzerland. Transfus Med Hemother 2016;DOI: 10.1159/000446264

42 Orton SL, Stramer SL, Dodd RY, Alter MJ: Risk factors for HCV infection among blood donors confirmed to be positive for the presence of HCV RNA and not reactive for the presence of anti-HCV. Transfusion 2004; 44:275-281.

43 Polizzotto MN, Wood EM, Ingham H, Keller AJ; Australian Red Cross Blood Service Donor and Product Safety Team: Reducing the risk of transfusion-transmissible viral infection through blood donor selection: the Australian experience 2000 through 2006. Transfusion 2008;48:55-63.

44 Van der Bij AK, Coutinho RA, Van der Poel CL: Surveillance of risk profiles among new and repeat blood donors with transfusion-transmissible infections from 1995 through 2003 in the Netherlands. Transfusion 2006;46:1729-1736

45 O'Brien SF, Chiavetta JA, Fan W, Xi G, Yi QL, Goldman M, Scalia V, Fearon MA: Assessment of a travel question to identify donors with risk of Trypanosoma cruzi: operational validity and field testing. Transfusion 2008;48:755-761.

46 El Ghouzzi MH, Boiret E, Wind F, Brochard C, Fittere S, Paris L, Mazier D, Sansonetti N, Bierling P: Testing blood donors for Chagas disease in the Paris area, France: first results after 18 months of screening. Transfusion 2010;50:575-583.

47 Grant IH, Gold JW, Wittner M, Tanowitz HB, Nathan C, Mayer K, Reich L, Wollner N, Steinherz L, Ghavimi F, et al: Transfusion-associated acute Chagas disease acquired in the United States. Ann Intern Med 1989; 111:849-851.

48 Nickerson P, Orr P, Schroeder ML, Sekla L, Johnston JB: Transfusion-associated Trypanosoma cruzi infec tion in a non-endemic area. Ann Intern Med 1989;111: 851-853.
49 Cimo PL, Luper WE, Scouros MA: Transfusion-associated Chagas' disease in Texas: report of a case. Tex Med 1993;89:48-50.

50 Leiby DA, Lenes BA, Tibbals MA, Tames-Olmedo MT: Prospective evaluation of a patient with Trypanosoma cruzi infection transmitted by transfusion. N Engl Med 1999;341:1237-39.

51 Cardo LJ, Asher L: Transfusion medicine illustrated. Electron micrographic study of the removal of Trypanosoma cruzi from blood products by leukodepletion filters. Transfusion 2006;46:1067-1068.

52 Young C, Losikoff P, Chawla A, Glasser L, Forman E: Transfusion-acquired Trypanosoma cruzi infection. Transfusion 2007;47:540-544.

53 Flores-Chávez M, Fernández B, Puente S, Torres P, Rodríguez M, Monedero C, Cruz I, Gárate T, Cañavate C: Transfusional chagas disease: parasitological and serological monitoring of an infected recipient and blood donor. Clin Infect Dis 2008;465:e44-47.

54 Stramer SL, Hollinger FB, Katz LM, Kleinman S, Metzel PS, Gregory KR, Dodd RY: Emerging infectious disease agents and their potential threat to transfusion safety. Transfusion 2009;49(suppl 2):1S-29S

55 Kessler DA, Shi PA, Avecilla ST, Shaz BH: Results of lookback for Chagas disease since the inception of donor screening at New York Blood Center. Transfusion 2013;53:1083-87.

56 Van Voorhis WC, Barrett LK, Eastman RT, Alfonso R, Dupuis K: Trypanosoma cruzi inactivation in human platelet concentrates and plasma by a psoralen (amotosalen $\mathrm{HCl}$ ) and long-wavelength UV. Antimicrob Agents Chemother 2003;47:475-479.

57 Singh Y, Sawyer LS, Pinkoski LS, Dupuis KW, Hsu JC Lin L, Corash L: Photochemical treatment of plasma with amotosalen and long-wavelength ultraviolet light inactivates pathogens while retaining coagulation function. Transfusion 2006;46:1168-1177.

58 Castro E, Gironés N, Bueno JL, Carrión J, Lin L, Fresno M: The efficacy of photochemical treatment with amotosalen $\mathrm{HCl}$ and ultraviolet A (INTERCEPT) for inactivation of Trypanosoma cruzi in pooled buffycoat platelets. Transfusion 2007;47:434-441.

59 Fearon MA, Scalia V, Huang M, Dines I, Ndao M, Lagacé-Wiens P: A case of vertical transmission of Chagas disease contracted via blood transfusion in Canada. Can J Infect Dis Med Microbiol 2013;24:32-34.

60 Seed CR, Kee G, Wong T, Law M, Ismay S: Assessing the safety and efficacy of a test-based, targeted donor screening strategy to minimize transfusion transmitted malaria. Vox Sang 2010;98:e182-192.

61 Schmidt M, Geilenkeuser WJ, Sireis W, Seifried E,Houfar K: Emerging pathogens - how safe is blood? Transfus Med Hemother 2014;41:10-17. 